\title{
Editorial
}

\section{Ignorant Humans, Smart Microbes}

\author{
Md. Zahirul Haque ${ }^{1}$
}

For centuries, man has searched for ways to obtain power beyond the mortal limits. That impulse for the ultimate, however, is not exclusively humans. Bugs too have it. And it seems that some of them can achieve this, at least, to some extent. These are the multidrug-resistant Gram-negative bacteria. These groups of bacteria, which scientists call the "superbugs," pose great risk to public health because they have developed resistance to make them impervious to powerful antibiotics of last resort.

In true sense, it's not a superbug per se, but a supergene. New Delhi metallo-beta-lactamases-1 (NDM-1), a genetic mutation, is the designation for carbapenemases that can be acquired by a variety of bacteria. Carbapenems are antibiotics of last resort against hardy infectants such as Escherichia coli and Klebsiella pneumoniae.

NDM-1 was first detected in a Klebsiella pneumoniae isolate from a Swedish patient of Indian origin in 2008. It was also found in enterobacteriaceae isolated from patients in the United Kingdom and elsewhere who have had healthcare contact in India or Pakistan.

The gene mutation NDM-1 was identified in 11 distinct bacterial species. To date, scientists have found 36 strains of Escherichia coli and 111 strains of Klebsiella pneumoniae containing NDM-1. NDM-1 has been isolated from Klebsiella pneumoniae, Escherichia coli, Citrobacter freundii, Enterobacter cloacae, and Morganella morganii. Most of these isolates carried the NDM1 gene in the bacterial plasmids. That makes these bacteria resistant to all antibiotics available today except to tigecycline and colistin.

A Cardiff University-led team found new strains of resistant bacteria in the Indian capital, including species which cause cholera and dysentery. The findings are the first evidence of the environmental spread of NDM-1, which had previously only been found in hospitals.

The researchers also find that the NDM-1 genes are much more prone to swap (by as much as 1 million times) between bacterial species at 30 degrees Celsius. This temperature happens to be within the daily reach for much of the year in many tropical countries like Bangladesh, including monsoon season, when floods and drain overflows are most likely, which potentially disseminates resistant bacteria.

Scientists in different part of the world are working for new family of antibiotics that can be used against these organisms. One is thiomarinol a molecule which can work as a new kind of antibiotic. But going from laboratory to pharmacy will take at least a decade that will effectively combat NDM-1, but scientists also note that the research needs to pick up now.

On December 11th 1945, at the end of his Nobel lecture, Alexander Fleming sounded a warning. Fleming could already see the future of antibiotic misuse. "There is the danger", he said, "that the ignorant man may easily underdose himself and by exposing his microbes to non-lethal quantities of the drug make them resistant." 
The virtual nonexistence of antibiotic policies and guidelines in developing countries to help doctors make rational choices with regard to antibiotic treatment is a major driver of the emergence and spread of multi-drug resistance. This is augmented by the unethical and irresponsible marketing practices of the pharmaceutical industry, and encouraged by the silence and apathy of the regulating authorities. Poor microbiology services in most parts of the world add to the problem. The problem is not only confined to people. Louise Slaughter, an American congresswoman who is also a microbiologist, calculates that four-fifths of the antibiotics used in America are given to livestock to get perfectly healthy animals. But it creates more opportunities for bacteria to evolve resistance.

With the emergence of these deadly variants, we are losing our ground in the war against infectious diseases, which was once thought to be over with the invention of very effective antimicrobials. Clinicians as well as microbiologist have a very important role in the prevention of spread of these dreaded multi-resistant pathogens across the world. They should actively participate to influence the policies by the government, develop guidelines for antibiotic therapy in their local hospitals, set up surveillance systems for drugresistant organisms, and educate healthcare workers and the general public about the dangers of multi-drug resistant organisms.

\section{References}

1. Walsh TR, Toleman MA, Poirel L, et al. Metallo- $\beta-$ lactamases: the quiet before the storm? Clin Microbiol Rev 2005; 18:306-25.

2. Kumarasamy KK, Toleman MA, Walsh TR, et al. Emergence of a new antibiotic resistance mechanism in India, Pakistan, and the UK: a molecular, biological, and epidemiological study. Lancet Infect Dis 2010;10:597-602.

3. Nordmann P, Cuzon G, Naas T. The real threat of Klebsiella pneumoniae carbapenemase-producing bacteria. Lancet Infect Dis 2009;9:228-36.

4. Zarfel G, Hoenigl M, Leitner E, et al. Emergence of New Delhi metallo- $\beta$-lactamase, Austria. Emerg Infect Dis 2011;17:129-30.

5. Bogaerts $P$, Verroken A, Jans $B$, et al. Global spread of New Delhi metallo- $\beta$-lactamase 1. Lancet Infect Dis 2010;10:831-2.

6. Mulvey MR, Grant JM, Plewes K, et al. New Delhi metallo- $\beta$-lactamase in Klebsiella pneumoniae and Escherichia coli, Canada. Emerg Infect Dis 2011;17:103-6. 